\title{
Resonant transport properties of tight-binding mesoscopic rings
}

\author{
Jingbo Li \\ Department of Physics, South China University of Technology, Guangzhou 510641, China \\ Zhao-Qing Zhang \\ Department of Physics, Hong Kong University of Science and Technology, Clear Water Bay, Kowloon, Hong Kong \\ Youyan Liu \\ CCAST (World Laboratory), P. O. Box 8730, Beijing 100080, China, \\ Department of Physics, South China University of Technology, Guangzhou 510641, China, \\ and Department of Physics, Hong Kong University of Science and Technology, Clear Water Bay, Kowloon, Hong Kong
}

(Received 19 June 1996)

\begin{abstract}
Using the tight-binding model, we have studied the resonant-transport properties of the multiply connected ring systems threaded by magnetic flux $\Phi$, and coupled to two electron reservoirs. For different flux $\Phi$ the electronic-energy spectrum and their corresponding total transmission probabilities of multiring systems have been calculated and compared. Some novel resonant-transport properties which display the Aharonov-Bohm effect have been found. We have also studied the same problem of a single ring with two barriers. It is found that for the high barriers the distribution of resonant states is coincident with the energy spectrum of the isolated ring, but the energies of resonant states have deviated from the eigenenergies of the system. [S0163-1829(97)11607-1]
\end{abstract}

\section{INTRODUCTION}

In the past decade developments in mesoscopic physics have made rapid progress. Quantum transport in the mesoscopic systems has been extensively studied both experimentally and theoretically during this period. ${ }^{1-23}$ For mesoscopic systems at very low temperatures, the scattering of phonons (dephasing scattering) is significantly suppressed and the phase coherence length of the electrons becomes large compared to the system dimensions, so that the systems can be modeled as phase coherent elastic scattering. Furthermore, if one considers the electron as a free particle, the idealized sample becomes an electron waveguide, which assumes that the electron transport through the system is perfectly ballistic. In recent years, there has been extensive work on the electronic properties of mesoscopic rings in the framework of the ballistic model or waveguide theory. ${ }^{16-20}$ Along this line, theoretical studies to date have concentrated largely on the isolated ring and open rings connected via leads to electron reservoirs, both of which are threaded by a magnetic flux. For the isolated ring, the persistent currents are the main subjects, in which an important and well-known conclusion is the "period halfing,", 22 i.e., the persistent current is periodic in magnetic flux $\Phi$ with a period $\Phi_{0} / 2$, $\Phi_{0}=h c / e$ being the elementary flux quantum. As for open ring systems the main interest is to study the relationship between the transmission coefficient $T$ and flux $\Phi$. In open ring systems, the electron reservoirs act as a source of energy dissipation or irreversibility, and all scattering processes in the leads and rings are assumed to be elastic. Along this line, to date the theoretical work is mostly based on waveguide theory to study the open single-ring or multiring systems.
$\mathrm{Xia}^{16}$ has studied the Aharonov-Bohm effect of the open single ring, i.e., by two leads the ring is connected with electronic reservoirs, by calculating the reflection and transmission amplitudes as functions of the magnetic flux, the arm lengths, and the wave vector. Deo and Jayannavar ${ }^{18,19}$ have studied the quantum transmission properties of serial stub or loop structures and the band formation in these geometric structure. Takai and Ohta ${ }^{20}$ have published a series of articles to investigate the same sort of problems but both magnetic flux and electrostatic potential are applied simultaneously. Before them, Cahay, Bandyopadhyay, and Grubin ${ }^{11}$ have studied the $A-B$-type conductance oscillation in the presence of either a magnetic flux or an electrostatic potential. On the other hand, $\mathrm{Wu}$ and Mahler ${ }^{15}$ have developed the quantum network theory of transport, by which the transmission probability for open $A-B$-type ring with arbitrary form factor has been studied in detail. For the tight-binding model, the advantages of which are that it is easier to induce disorder and there is a definite energy band, however, there are only a few papers devoted to investigating the transport properties of single ring system by the use of the Green's function. ${ }^{17}$ We have studied a mesoscopic ring system connected in parallel, and found that the transmission coefficient $T$ is periodic in flux with a period $2 \Phi_{0}$, but not $\Phi_{0}$ as that of the double-ring systems connected in series. ${ }^{23}$ To the best of our knowledge, in the framework of the tight-binding model there is no work devoted to study the transport properties of multiring systems connected in serial. Especially the resonant transport phenomenon appearing in a variety of incident electronic energy $E$ and magnetic flux $\Phi$ has not been reported.

In this paper, based on the tight-binding model and by the 
use of scattering matrix, we concentrate on studying the serially connected ring systems, which are composed of onedimensional ordered chains. Of main interest to us is to investigate the relationship among the resonant states, the energy spectrum of the closed-ring system and magnetic flux. In a general picture, if the energy of the incident electron is coincident with the eigenenergy of the energy spectrum, the electron would easily transmit the system and form a resonance peak. On the other hand, the Aharonov-Bohm effect would influence this transmission. Therefore, it would be very interesting to see a $T-E-\Phi$ three-dimensional transmission diagram which displays the influence of flux $\Phi$ on the resonant transmission behavior. It is found that roughly the resonant states have the same structure as the energy spectrum of the serially connected ring systems, but when levels of the spectrum are compared the resonant energies have shifted; besides that, all of the states closed to flux $\Phi_{0} / 2$ are antiresonant ones. Our numerical results for the open multiring systems, as mentioned above, do not show a very good agreement between the resonance peaks and eigenenergies. Therefore, to expect a better agreement we have investigated an open single ring with two site barriers by setting the site energy $\varepsilon_{b}$ much higher than that of ideal ordered chain, which composes the leads and ring. We have found that, when the barriers are high enough, the number of resonant states is coincident with the number of levels of isolated ring, but the energies of resonant states have slightly shifted toward the center of spectrum and the resonant states closed to $\Phi=\Phi_{0} / 2$ are still missed. For these transport behaviors we will give a plausible explanation. This paper has been organized as follows. In Sec. II we state the theoretical treatment for calculating the transmission coefficients of the mesoscopic ring systems studied in this article, including multiring systems and single ring with two barriers. In Sec. III we present the results of calculation and their physical explanation. Section IV is a brief summary.

\section{TRANSMISSION COEFFICIENTS OF THE MESOSCOPIC RING SYSTEMS}

\section{A. Transmission amplitude of a single ring}

For an open single ring, i.e., a ring connected via two leads to electron reservoirs, we assume that the leads and ring are composed of one-dimensional ordered chains with site energy $\varepsilon_{n}$, transfer integral $J$ between nearest neighbor sites. If we denote the electron energy by $E$, and wave function at the $n$th site by $\phi_{n}$, the tight-binding equations are

$$
\left(\varepsilon_{n}-E\right) \phi_{n}=\sum_{n^{\prime}} J_{n, n^{\prime}} \phi_{n+n^{\prime}},
$$

where the sum runs over the nearest neighbors of $n$ site. In this paper we choose the site energy $\varepsilon_{n}=0$ for ordered chain.

Referring to Fig. 1(a), for a single ring threaded by flux $\Phi$, and connected via leads to electron reservoirs, the reflection and transmission amplitudes can be given as flollows: ${ }^{14,23}$

$$
r=e^{2 i q N}\left\{c-\frac{2 K}{d}\left[b \cos \phi+a-e^{i \psi}\left(b^{2}-a^{2}\right)(b-a)\right]\right\},
$$

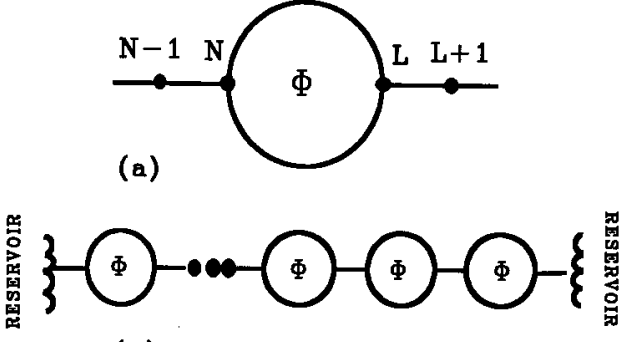

(b)

FIG. 1. (a) A single ring coupled to two electron reservoirs via two ordered leads. (b) Serially connected mesoscopic rings threaded by magnetic flux $\Phi$, and coupled to two electron reservoirs via ideal leads.

$$
t=2 K \frac{\cos (\phi / 2)}{d}\left[(b-a)^{2}-e^{-i \psi}\right],
$$

where

$$
\begin{gathered}
\phi=2 \pi \Phi / \Phi_{0}, \\
\psi=2 q(L-N)=q S, \\
a=e^{-2 i q N}\left[\frac{2 i J \sin q}{D}-1\right], \\
b=e^{-2 i q N} \frac{2 i J \sin q}{D}, \\
c=e^{2 i q N}\left[\frac{2 i J \sin q}{D}-1\right], \\
\sqrt{K}=\frac{2 i J \sin q}{D}, \\
D=E-\varepsilon_{n}+3 J e^{i q}, \\
q=\arccos \left(-\frac{E-\varepsilon_{n}}{2 J}\right), \\
d=2 b^{2} \cos \phi-e^{-i \psi}-\left(b^{2}-a^{2}\right)^{2} e^{i \psi}+2 a^{2} .
\end{gathered}
$$

The $N$ and $L$ are the left site and right site, through which the ring is coupled to the reservoirs via leads as shown in Fig. 1(a), and $S$ is the circumference length of the ring. In the next paragraph, we disscuss how to use these single-ring formulas to treat the multiring systems.

\section{B. Transmission amplitude of $N$ connected rings}

In order to obtain the total transmission coefficient of $N$ connected rings, we divide the studied system into two subsystems at site $n$, which usually is the central site of lead connected two neighbor rings, referred to as the left subsystem and the right subsystem. In this paper, $t$ and $r$ represent the transmission and reflection amplitudes of the incident wave from the left; while $t^{\prime}$ and $r^{\prime}$ represent the transmission and reflection amplitudes of the wave from the right. We now denote the transfer matrix of the studied system by $\tau$; in general, $\tau$ can be expressed as ${ }^{14}$ 


$$
\tau=\frac{1}{t^{\prime}}\left(\begin{array}{cc}
t t^{\prime}-r r^{\prime} & r^{\prime} \\
-r & 1
\end{array}\right)
$$

If we denote the transfer matrix of the left subsystem by $\tau_{L}$, and the right one by $\tau_{R}$, then, the transfer matrix $\tau$ of whole system is equal to $\tau_{R} \tau_{L}$,

$$
\tau=\tau_{R} \tau_{L}
$$

Inserting Eq. (4) into Eq. (5), we can obtain the total transmission and reflection amplitudes expressed by $t_{R}, t_{L}$, $t_{R}^{\prime}, t_{L}^{\prime}, r_{R}, r_{L}, r_{R}^{\prime}, r_{L}^{\prime}$, and the formulas are

$$
t=\frac{t_{R} t_{L}}{1-r_{R} r_{L}^{\prime}}, \quad r=\frac{\left(t_{L} t_{L}^{\prime}-r_{L} r_{L}^{\prime}\right) r_{R}+r_{L}}{1-r_{R} r_{L}^{\prime}} .
$$

It is important and easy to derive that the relationships between $t$ and $t^{\prime}, r$ and $r^{\prime}$ are

$$
t^{\prime}=t, \quad r^{\prime}=r e^{-2 i q(N+L)},
$$

where $N$ and $L$ are the left and right site of the ring, respectively, as shown in Fig. 1(a). For a serially connected doublering system, if the left ring is referred to as the left subsystem, and other one as right subsystem, then in terms of formulas (2), (3) and (6), we can calculate the total transmission and reflection amplitudes of double-ring systems. In the same way, if we take a two-ring system as right subsystem, and add a single ring from the left as the left subsystem, again by the use of above procedure, we can obtain the total transmission and reflection amplitudes of three-ring systems by repeatedly using the formula (6). Following the above procedure, if we have obtained the $(N-1)$-ring systems and referred it as right subsystem; then adding a single ring from the left as the left subsystem, by the same calculation procedure we can obtain the total transmission and reflection amplitudes of $N$-ring systems. The transmission and reflection coefficients $T$ and $R$ are given by the absolute square of the corresponding amplitudes, and satisfy the conservation condition $T+R=1$. These calculation processes for arbitrary multiring systems are easy to be handled with a computer.

Figure 1(b) shows a serially connected $N$-ring system threaded by same magnetic flux $\Phi$, and coupled to two electron reservoirs via idea leads. In our calculation program, in principle, the transmission coefficient of arbitrary number of connected rings can be calculated.

\section{A single ring with two barriers}

For the purpose of investigating the relationship between the resonant states and the eigenenergies of isolated ring, we have studied a single ring with two barriers at sites $n_{b}=N$ -1 and $L+1$ with the site energy $\varepsilon_{b}>\varepsilon_{0}=0, \varepsilon_{0}$ being the site energy of ideal leads and ring, as shown in Fig. 1(a). Because of the site energy $\varepsilon_{n}$ being simply the energy of the atomic level, the site with high site energy $\varepsilon_{b}$ becomes an ideal barrier. By the use of the transfer matrix, after a long but straightforward calculation, we have obtained the transmission and reflection amplitudes of the present system as follows:

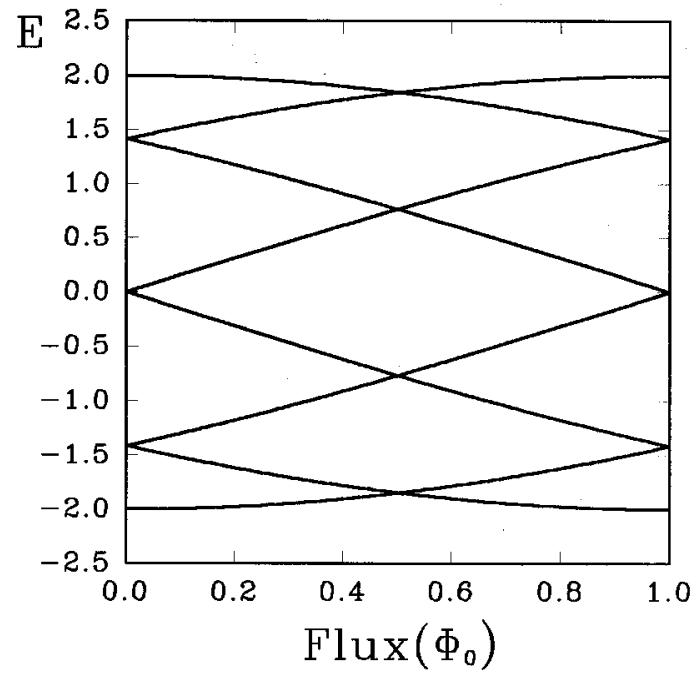

FIG. 2. Energy spectrum of isolated single ring as a function of magnetic flux $\Phi$ for the tight-binding model. The parameters of the system are site energy $\varepsilon_{n}=0$, transfer integal $J=1.0$; the site number of the ring is 8 .

$$
\begin{gathered}
t=\frac{2 i J \sin q}{E-\varepsilon_{b}+2 J e^{i q}}, \\
r=\frac{-\left[\left(E-\varepsilon_{b}\right)+2 J \cos q\right] e^{2 i q n_{b}}}{E-\varepsilon_{b}+2 J e^{i q}} .
\end{gathered}
$$

In this paper we choose $J=1.0$, therefore the range of incident electron energy is from -2.0 to 2.0. The incident electron with any energy from -2.0 to 2.0 will be decayed by the barrier site $n_{b}$. In this case, the ring, in some sense, can be analogous to a double-potential well. The resonant states of incident electrons can be expected to possess the eigenenergies of the isolated ring.

\section{THE NUMERICAL RESULTS AND DISCUSSION}

\section{A. Electronic energy spectrum of ring systems}

In order to investigate the relationship between the resonant states and the electronic energy spectrum of ring systems, the numerical exact diagonalization method has been used to calculate the energy spectrum of the studied systems. For the isolated ring-systems threaded by flux $\Phi$, the Hamiltonian simply is

$$
H=\sum_{n}|n\rangle \varepsilon_{n}\left\langle n\left|+\sum_{n \pm 1}\right| n\right\rangle J_{n, n \pm 1}\langle n \pm 1|,
$$

where $\varepsilon_{n}$ is the site energy, and $J_{n, n \pm 1}$ is the transfer integral, which equals $J e^{ \pm i 2 \pi \Phi /\left(\Phi_{0} S\right)}$, where $S$ is the circumference length of the ring. ${ }^{21}$

The energy spectra of an isolated one-ring system and three-ring systems connected to each other by ideal leads with length being a lattice constant are shown in Fig. 2 and Fig. 3, respectively, and each ring has eight sites. They show that the electronic eigenenergy $E$ is periodic in flux $\Phi$ with a period $\Phi_{0}, \Phi_{0}$ being the elementary magnetic flux. In the case of $\Phi=0$, the electronic states are degenerated at ener- 


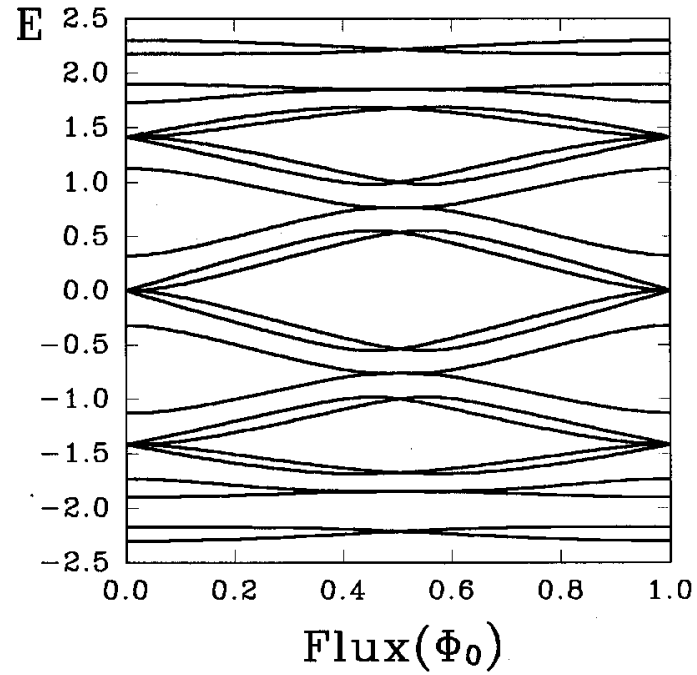

FIG. 3. Same as Fig. 2 for a serially connected three-ring system. The distance of neighbor rings is a lattice constant.

gies $E=0.0$ and $E \cong \pm 1.41421$, as shown in Fig. 2 and Fig. 3 . As magnetic flux $\Phi$ increases from zero, the degenerate states split due to the magnetic Aharonov-Bohm effects. When the flux $\Phi$ reaches $\Phi_{0} / 2$, the electronic states degenerate again. The whole diagram shows a symmetric pattern to $\Phi=\Phi_{0} / 2$ and $E=0$. Comparing Fig. 3 with Fig. 2 we can see that the energy spectrum of three-ring systems has same structure as the single ring; our calculation has shown that the fundamental configuration of the energy spectrum does not change as the number of connected rings increases.

\section{B. Transport properties of serially connected ring systems}

Formula (6) displays that the transmission coefficient $T=|t|^{2}$ of the ring systems connected with electron reservoirs has a very heavy and complicated expression; it is necessary to use computer to obtain the numerical results. For guaranteeing the accuracy of numerical calculation, in every stage after obtaining the transmission and reflection coefficients, we have checked the results by the criterion $|t|^{2}+|r|^{2}=1$. Our numerical accuracy reaches to $10^{-14}$, which adequately guarantees that the calculation is reliable. For simplicity, we have assumed that all of the rings are identical. Applying formula (6) repeatedly, we can easily obtain the transmission coefficient $T$ for a serially connected ring systems. We have calculated a series of multiring systems with ring number $N=1,2,3,4,5,6,8,10$, respectively. As typical cases, we display the results of single-ring and threering systems in Fig. 4 and Fig. 5.

We have noticed that in the framework of waveguide theory for the mutiply connected ring systems, in some case the frequency of quantum oscillation is proportional to the number of connected rings. However, for the tight-binding model used in the present paper in all cases with different $N$, one cannot find the above proportional properties. The resonant states exhibit a very complicated behavior following the change of electron energy and magnetic flux. We found that, in general, more anomalous peaks appear as the number of connected rings increase, but the corresponding

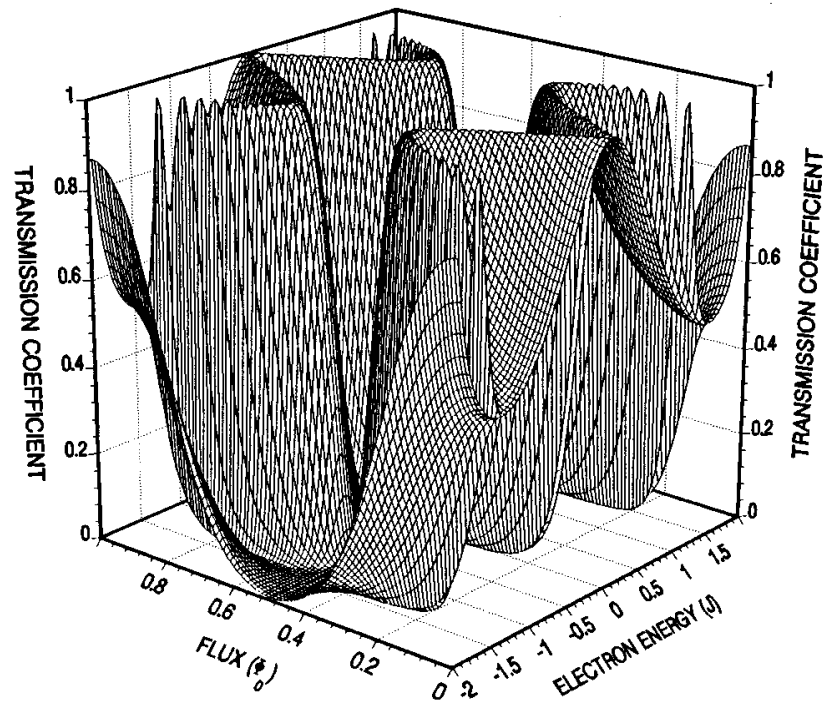

FIG. 4. Transmission coefficient $T$ versus magnetic flux $\Phi$ and incident electron energy $E$ for a single ring connected to two electron reservoirs. All the relative parameters are same as those of Fig. 2.

between the frequency of quantum oscillation and the number of connected rings is not explicit. From Figs. 4 and 5 we can see that the rule of apearance of resonance peaks cannot be easily tackled, but for the waveguide ring systems it can. ${ }^{20}$ The reason for this is that, for the waveguide theory, the electron transport through the system is perfectly ballistic, so that it may be analogous to serial superlattices. ${ }^{18,20}$ However, our numerical calculation shows that for the tight-binding model there is no such analogy. The above difference between the wave guide theory and tight-binding calculation mainly is due to such a fact that for the tight-binding model there is additional scattering in the junction, the combination of which with the multibackscattering between the neighbor

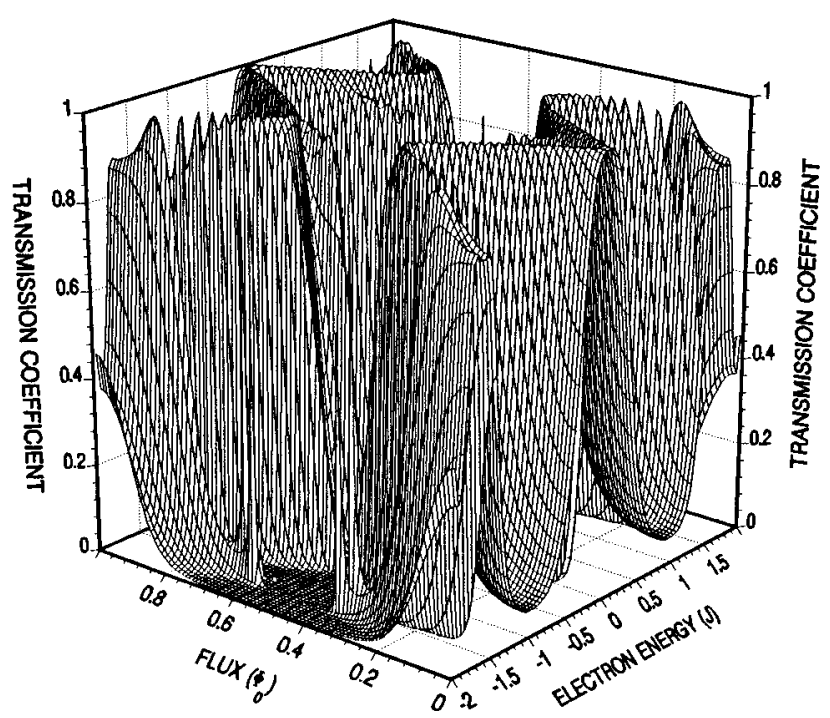

FIG. 5. Same to Fig. 4 for a serially connected three-ring system. The distance of neighbor rings is a lattice constant. 


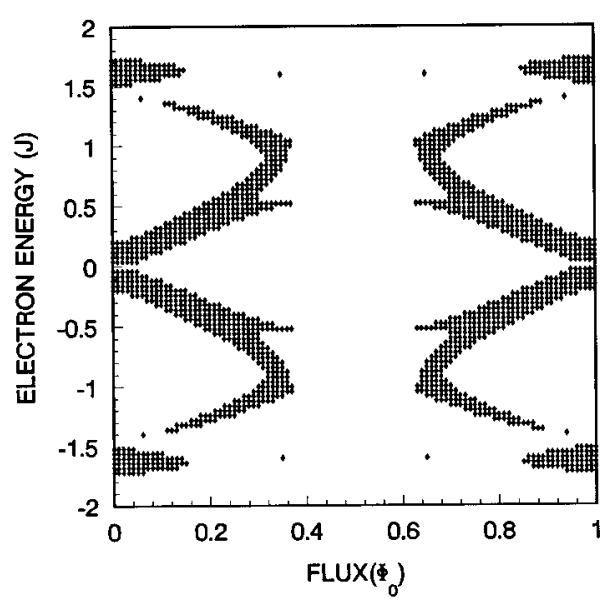

FIG. 6. Resonance spectrum of Fig. 5 is shown in the electron energy versus magnetic flux plane. The dots represent the incident electron states with total transmission coefficient $T \geqslant 0.85$. Aharonov-Bohm effect results in the missing of resonance peak in the region closed to $\Phi=\Phi_{0} / 2$ (see text).

rings gives rise to different results from the waveguide theory. The bigger the ring number is, the more different the transport behavior will be.

From Fig. 4 and Fig. 5, it is noteworthy that all the resonance peaks appear along a sinuouslike curve, ${ }^{23}$ which corresponds another kind of quantum oscillation behavior. Because to date all previous work based on the waveguide theory has not shown a $T-E-\Phi$ three-dimensional diagram, therefore, at the moment we cannot give a detailed comparison for these two different models. In the next paragragh, we will explain that the location of these resonance peaks results from the competition between the Aharonov-Bohm magnetic effect and resonance effect existing in the degenerate states.

Now we discuss the relationship between the resonance peaks and the energy spectrum of the studied systems in the presence of the Aharonov-Bohm flux threading the rings. For the single ring, from Fig. 4 we can easily see that the resonant states distribute in coincidence with the energy spectrum of the single ring, as shown in Fig. 2, except in the region of the diagram closed to $\Phi=\Phi_{0} / 2$. In all of the $T$ $E$ - $\Phi$ diagrams we have observed a common fact that in the region closed to $\Phi_{0} / 2$ the transmission coefficient is always equal to zero. This conclusion also can be reached from formula (3), which implies that when $\Phi=\Phi_{0} / 2$ transmission amplitude $t=0$. This is a typical Aharonov-Bohm effect, because in the present case the upper and bellow arms are identical, when $\Phi=\Phi_{0} / 2$, the accummulated magnetic phases through upper and bellow arms at site $L$ of Fig. 1(a) are $\pm 2 \pi \Phi / \Phi_{0}= \pm \pi$, respectively, so that the electron wave attenuates to zero because of the interference effect. But if one compares Fig. 5, corresponding to three-ring system, with Fig. 4, one can see that the distribution of transmission coefficient is further complicated. For better visualization we plot those points of Fig. 5, of which the transmission coefficient is not less than $0.85(T \geqslant 0.85)$, and shown in Fig. 6 . We can consider that this picture displays the distribution of resonance peaks. Compared with Fig. 3, which displays the energy spectrum of the three-ring system, it is found that

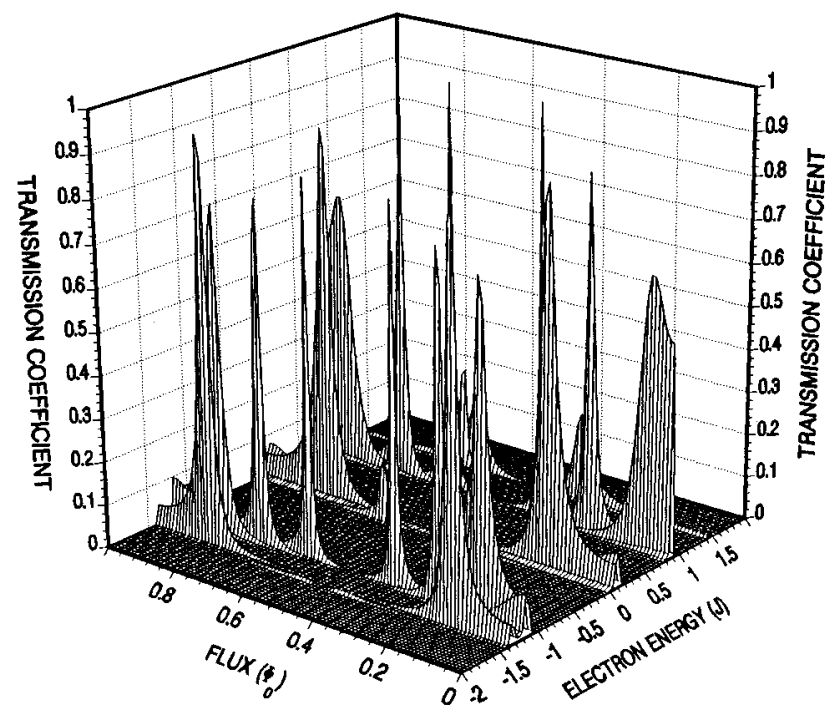

FIG. 7. $T-E-\Phi$ diagram for an open single ring with two barriers as shown in Fig. 1(a), the site energy $\varepsilon_{b}=20.0$ at the $N-1$ and $L+1$ sites, energy interval $\Delta E=0.04$. Compared with Fig. 4 the resonance peaks are much sharper and sparser.

when the energy of incident electron is closed to one of the eigenenergies of the system, the electron will easily transport through the system and the transmission coefficient exhibits a resonance peak. This result is consistent with that of Ref. 4, in which the quantum oscillation of a single ring was studied. As we have mentioned, there exists an energy deviation between the resonance peak and the energy spectrum of isolated ring systems. This point can be seen also from a comparison between Fig. 3 and Fig. 6. The deviation should be due to the additional junction scattering when the ring is connected via leads to electron reservoirs. Our numerical calculation displays that the junction scattering results in two effects, firstly it depresses the peaks at $E= \pm 2$, and secondly, it shifts the peak positions if compared with the energy spectrum of isolated ring systems. From Figs. 2 and 3 one can see that in the absence of magnetic flux the electronic states are degenerate. When the magnetic field is applied, the time-reversal symmetry is broken and the degenerate states split into 2 . On the other hand, if the number of rings increases, the corresponding level proportionally increases. From these two facts one would expect that following the increase of ring number and magnetic flux the number of peaks would proportionally increase as in the case of waveguide theory. ${ }^{20}$ But in our tight-binding model calculation even the energy step is taken by $10^{-3}$ we still cannot observe this kind of result, the peaks increase, but not proportionally.

\section{Resonant transport properties of a single ring with two barriers}

This paragraph is devoted to investigating the idea of whether or not in some cases the studied ring systems can be analogous to a double-potential well, so that the resonance peaks, no matter what their location or number, should be coincident with the spectrum of isolated ring systems. For 


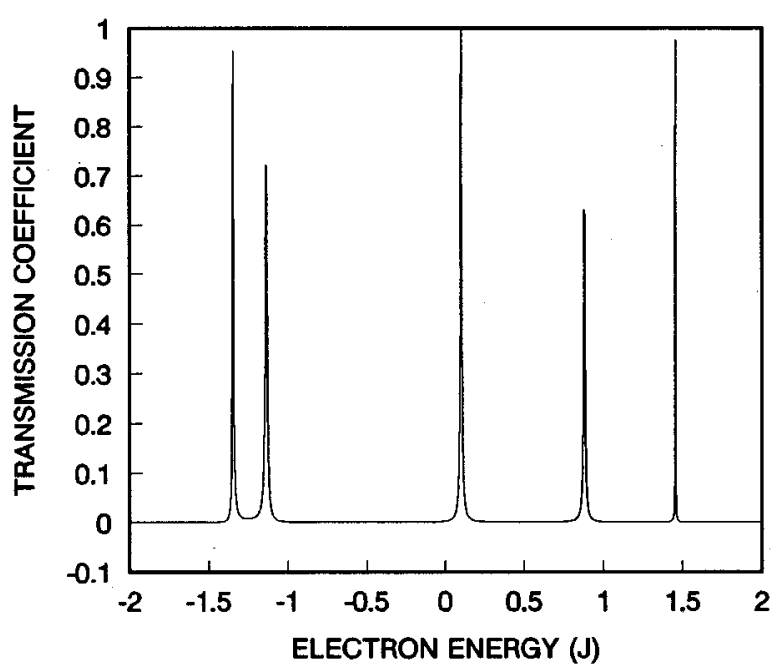

FIG. 8. A cross section of Fig. 7 with $\Phi=0$ and the energy interval $\Delta E=0.001$. There are five resonance peaks, which are coincident with the number of eigenenergies of energy spectrum shown in Fig. 2. Barriers and junction scattering have deviated very much from the resonance peak energies from the corresponding eigenenergies of the isolated single ring.

this purpose, we have investigated an open single ring with two barriers as shown in Fig. 1(a). By the use of formulas (8) and (9), we have calculated the $T-E-\Phi$ diagram for the system with barrier site energy $\varepsilon_{b}=20.0$, and the results are shown in Fig. 7. If we compare it with Fig. 4, which corresponds to same single-ring system but without barrier sites, we can see that the resonance peak behavior is quite different. It turns out to be much more sparse and sharp, and also better corresponds to the spectrum of the isolated ring. For observing this point, in Fig. 8 and Fig. 9 we plot the transmission coefficient $T$ of the system versus incident electronic energy $E$ for $\Phi=0$ and $\Phi=0.38 \Phi_{0}$, respectively. Their resonance peak numbers are coincident with that of eigenenergies of energy spectrum. Besides that, we have also noticed some features which appear due to the existence of barriers. Firstly, we found that both pictures of Fig. 8 and Fig. 9 are not symmetric again to $E=0$ for the incident electronic energy $E$, because the two barriers with $\varepsilon_{b} \neq 0$ have changed the symmetry of incident electronic energy to $E=0$. Secondly, in the absence of magnetic field, as shown in Fig. 2, there are three degenerate peaks of the transmission coefficient. When the magnetic field is applied, these degenerate states split, which causes three additional resonance peaks appearing in Fig. 9. The physical origin of these increasing resonance peaks lays on that the magnetic flux in the ring gives a contrary phase shift, $e^{i \theta}$ in the upper arm and $e^{-i \theta}$ in the lower one, and the contrary phase shift causes the degenerate peak splitting. Thirdly, from the energy spectrum shown in Fig. 2, we can see that at the point $\Phi=0$ and $\Phi=0.38 \Phi_{0}$ there are five and eight eigenenergies; correspondingly, in Fig. 8 and Fig. 9, five and eight resonance peaks appear, respectively. If comparing the energies, we will find that some resonant states deviate from the eigenenergies of the ring system. A plausible explanation is that first the additional barriers change the energy spectrum of the whole system; on the other hand, the barriers also enhance

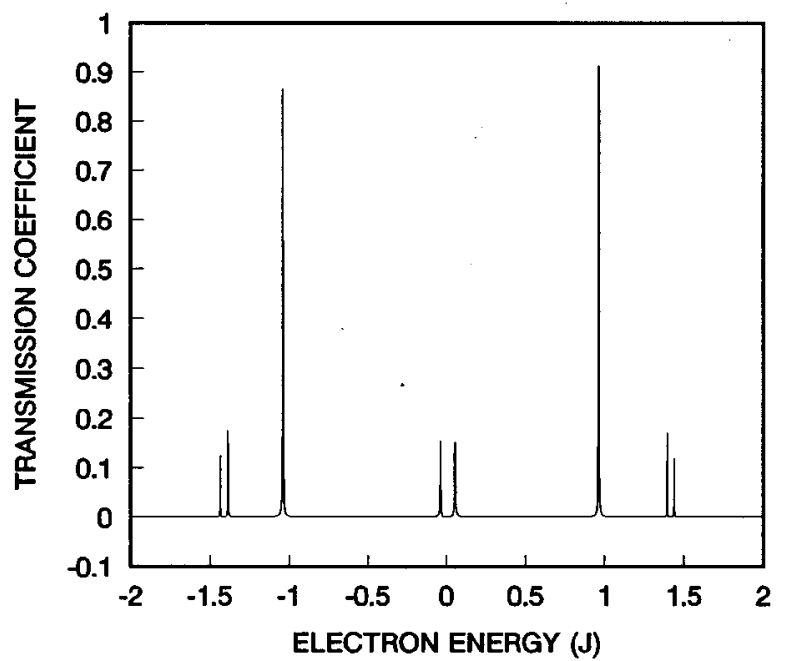

FIG. 9. Same as Fig. 8, but flux $\Phi=0.38 \Phi_{0}$. The number of resonance peaks is in agreement with Fig. 2, but there is also a deviation of energy as in the $\Phi=0$ case.

the scattering of the studied system for the incident electrons. These two facts result in the deviation of resonant energies from eigenenergies of studied system.

The experimental plausibility of the theoretical results is an interesting problem for which Takai and $\mathrm{Ohta}^{20}$ have given a detailed discussion [Phys. Rev. B 50, 11132 (1995)]. In principle, their discussion is also suitable for the tightbinding model, even though they deal with a waveguide model. The experimental results definitely depend on the geometric cofiguration of the system. For a chosen cofiguration, the measurement of the $T-E-\Phi$ spectrum is meaningful work, which can serve to adjudge the correctness of theory, and also would give some suggestion for future theoretical study.

\section{SUMMARY}

We have studied the electron transport properties of serially connected mesoscopic ring systems in the framework of the tight-binding model. Including the magnetic AharonovBohm effect, firstly the transmission amplitude of a single ring is obtained by the scattering matrix of the three-terminal splitter. The transmission coefficient of $\mathrm{N}$-connected ring systems is derived by the treatment of adding a single ring from the left side, one by one. The energy spectrum of the studied systems is calculated by the numerical diagonalization method. In comparison with the results of the waveguide model, some new electron transport properties are found. We have found that more anomalous peaks of transmission coefficient appear as the number of connected rings increases, and the behavior of the quantum oscillation is much complicated, even though a fundamental structure of the resonance spectrum is kept. But in contrast with waveguide results, the frequency of quantum oscillation is not proportional to the number of connected rings. The transmission coefficient of the system depends on the energy of the incident electron as well as the magnetic flux penetrating the rings. The distribution of resonance peaks is similar to the energy spectrum of the studied systems, i.e., if the energy of the incident electron is closed to the eigenenergy of the system, the incident elec- 
tron is easy to transport through the system. For the single ring with two barriers, we have found that it is the two barriers that give rise to the deviation of the energies of resonant states from the eigenenergies of the isolated ring. Most of the above transport properties have been explained by the Aharonov-Bohm magnetic effect and the additional scattering of the three-terminal junction.

\section{ACKNOWLEDGMENTS}

This work was supported by the National Natural Science Foundation of China and GuangDong Provincial Natural Science Foundation of China as well as DAG grant of Hong Kong University of Science of Technology, Grant No. 94/95 SC14.
${ }^{1}$ R. Landauer, Philos. Mag. 21, 863 (1970).

${ }^{2}$ H. L. Engquist and P. W. Anderson, Phys. Rev. B 24, 1151 (1981).

${ }^{3}$ M. Büttiker, Y. Imry, and R. Laudauer, Phys. Lett. A 96, 365 (1983).

${ }^{4}$ M. Büttiker, Y. Imry, and M. Y. Azbel, Phys. Rev. B 30, 1982 (1984).

${ }^{5}$ Y. Gefen, Y. Imry, and M. Y. Azbel, Phys. Rev. Lett. 52, 129 (1984).

${ }^{6}$ M. Büttiker, Y. Imry, R. Landauer, and S. Pinhas, Phys. Rev. B 31, 6207 (1985).

${ }^{7}$ M. Heiblum, M. Nathan, D. Thomas, and C. Knoedler, Phys. Rev. Lett. 55, 2200 (1985).

${ }^{8}$ U. Sivan and Y. Imry, Phys. Rev. B 33, 551 (1986).

${ }^{9}$ C. Umbach, C. Van. Haesendonck, R. Laibowitz, S. Washburn, and R. Webb, Phys. Rev. Lett. 56, 386 (1986).

${ }^{10}$ O. Entin-Wohlman, C. Hartzstein, and Y. Imry, Phys. Rev. B 34, 921 (1986).

${ }^{11}$ M. Cahay, S. Bandyopadhyay, and H. L. Grubin, Phys. Rev. B 39, 12989 (1989).

${ }^{12}$ L. P. Levy, G. Dolan, J. Dunsmuir, and H. Bouchiat, Phys. Rev. Lett. 64, 2074 (1990).
${ }^{13}$ P. A. Lee, A. D. Stone, and H. Fukuyama, Phys. Rev. B 35, 1039 (1990).

${ }^{14}$ D. Kowal, U. Sivan, O. Entin-Wohlman, and Y. Imry, Phys. Rev. B 42, 9009 (1990).

${ }^{15}$ C. H. Wu, G. Mahler, Phys. Rev. B 43, 5012 (1991).

${ }^{16}$ J. Xia, Phys. Rev. B 45, 3593 (1992).

${ }^{17}$ A. Aldea, P. Gartner, and I. Corcotoi, Phys. Rev. B 45, 14122 (1992).

${ }^{18}$ P. Singha Deo and A. M. Jayannavar, Phys. Rev. B 50, 11629 (1994).

${ }^{19}$ A. M. Jayannavar and P. Singha Deo, Phys. Rev. B 51, 10175 (1995).

${ }^{20}$ D. Takai and K. Ohta, Phys. Rev. B 48, 1537 (1993); 50, 18250 (1994); 50, 2685 (1994); 50, 11132 (1995).

${ }^{21}$ Weimin Zhang, Wenji Deng, and Youyan Liu, Chin. Phys. Lett. 11, 357 (1994).

${ }^{22}$ Wenji Deng, Youyan Liu, and Changde Gong, Phys. Rev. B 50, 7655 (1994).

${ }^{23}$ Youyan Liu, Honglin Wang, Zhao-Qing Zhang, and Xiujun Fu, Phys. Rev. B 53, 6943 (1996). 